\title{
INFINITE-DIMENSIONAL METHODS IN FINITE-DIMENSIONAL GEOMETRIC TOPOLOGY
}

\author{
BY ROSS GEOGHEGAN ${ }^{1}$ AND R. RICHARD SUMMERHILL
}

Communicated by R. D. Anderson, May 22, 1972

1. Introduction. ${ }^{2}$ We use the methods of infinite-dimensional topology to derive new information about the topology of euclidean spaces and manifolds. The idea is to partition euclidean $n$-space $E^{n}$ into a $k$-dimensional pseudo-boundary $(0 \leqq k<n)$ and an $(n-k-1)$-dimensional pseudo-interior, and to deduce negligibility theorems analogous to those known for the pseudo-boundary and the pseudo-interior (denoted by s) of the Hilbert cube $I^{\omega}$. Since $s$ is homeomorphic to Hilbert space $l_{2}$, there is a sense in which we are giving the correct finite-dimensional analogues of $l_{2} \quad$ (see $\left.\S 5\right)$.

DEFINITION. A subset $X$ of a metric space $Y$ (with metric $d$ ) is strongly negligible in $Y$ if, for each open set $U$ in $Y$ and each map $\varepsilon: U \rightarrow R^{+}$, there is a homeomorphism $h: Y \rightarrow Y-(X \cap U)$ fixing $Y-U$ such that $d(x, h(x))<\varepsilon(x)$ for all $x \in U$. This is a topological property independent of $d$.

THEOREM 1.1. $E^{n}$ is the union of two disjoint dense subsets $B^{k}$ and $P^{n-k-1}$ such that (1) if $n \leqq 2 k+1$, any $\sigma$-compact subset of $P^{n-k-1}$ is strongly negligible in $P^{n-k-1}$, and (2) if $n \geqq 2 k+1$, any compact subset of $B^{k}$ is strongly negligible in $B^{k}$. If $n=2 k+1$, any $k$-dimensional compactum can be embedded in $B^{k}$ or in $P^{k}$.

Notation. Superscripts on spaces, e.g., $B^{k}, P^{n-k-1}$, indicate dimension.

We call $B^{k}$ of Theorem 1.1 the universal $k$-dimensional pseudo-boundary of $E^{n}$. It is built out of Menger universal compacta [13], [17]. (See §3.) $P^{n-k-1}$ of Theorem 1.1 is the corresponding pseudo-interior.

Another kind of $k$-dimensional pseudo-boundary in $E^{n}$ can be built out of polyhedra as follows.

Let $J_{0}$ be a rectilinear PL triangulation of $E^{n}$, all $n$-simplexes having the same diameter. Let $J_{i} \quad(i \geqq 1)$ be the $i$ th barycentric subdivision of $J_{0}$, its $k$-skeleton being $J_{i}^{k}$. The polyhedral $k$-dimensional pseudo-boundary of $E^{n}$ is $\widetilde{B}_{n}^{k}=\bigcup_{i=1}^{\infty}\left|J_{i}^{k}\right|$. The corresponding pseudo-interior is $\widetilde{P}_{n}^{n-k-1}=$ $E^{n}-\widetilde{B}_{n}^{k}$.

AMS 1970 subject classifications. Primary 57A15; Secondary 57A20.

${ }_{1}^{1}$ Supported by NSF grant GP-7952X3.

2 This is a summary of a paper entitled Pseudo-boundaries and pseudo-interiors in euclidean spaces and topological manifolds. 
TeRminology. A polyhedron is a space homeomorphic to the body of a countable locally finite simplicial complex; a subpolyhedron of $E^{n}$ is the body of such a complex when linearly embedded in $E^{n}$; a polyhedron $X$ in $E^{n}$ is tame if there is a homeomorphism $h$ of $E^{n}$ such that $h(X)$ is a subpolyhedron of $E^{n}$.

THEOREM 1.2. (1) Any subpolyhedron of $E^{n}$ of dimension $\leqq n-k-1$ can be embedded in $\widetilde{P}_{n}^{n-k-1}$ so as to be tame in $E^{n}$; (2) if $n \leqq 2 k+1$, $n \neq 4$, then the countable union of polyhedra, each tame in $E^{n}$ and lying in $\widetilde{P}_{n}^{n-k-1}$, is strongly negligible in $\widetilde{P}_{n}^{n-k-1}$; (3) if $n \geqq 2 k+1, n \neq 4$, then any compact subset of $\widetilde{B}_{n}^{k}$ is strongly negligible in $\widetilde{B}_{n}^{k}$; (4) if $0 \leqq k \leqq$ $n-3, k \neq 1, n \neq 4$, then the countable union of compact polyhedra of dimension $\leqq k$ in $\widetilde{P}_{n}^{n-k-1}$ is strongly negligible in $\widetilde{P}_{n}^{n-k-1}$; (5) any $k$ dimensional polyhedron can be embedded in $\widetilde{B}_{2 k+1}^{k}$ or in $\widetilde{P}_{2 k+1}^{k}$.

In all of these constructions the analogy with $I^{\omega}$ is striking. It is explained in $\$ 5$.

The theory of negligibility is briefly set out in $\$ 2$. It is then applied in $\$ \$ 3$ and 4. The analogy with $I^{\omega}$ is discussed in $\S 5$. The extension to manifolds is in $\S 6$. Theorem 1.1 follows from the ideas of $\S 3$ and Theorem 1.2 from those of $\S 4$.

2. Pseudo-boundaries in complete metric spaces. Given a complete metric space $Y$, when can $Y$ be partitioned into a set $B$ (analogous to the pseudo-boundary of $I^{\omega}$ ) and a set $P$ (analogous to the pseudointerior of $I^{\omega}$ ) so that "tame" subsets of $B$ or $P$ are negligible and what constitutes a family of "tame" sets? The answer, Theorems 2.1, 2.2, and 2.3, is a variation on an idea of Torunczyk [18] which in turn is based on work of Anderson [1] and Bessaga-Pelczynski [3]. See also [19].

DEFINITIONS AND NOTATION FOR $\$ 2 . H(Y)$ is the set of homeomorphisms of $Y$. If $U$ is open in $Y, \varepsilon: U \rightarrow R^{+}$is a map and $f \in H(Y)$, let

$$
\begin{aligned}
& V_{U}(f, \varepsilon)=\left\{h \in H(Y) \mid d(f(y), h(y))+d\left(f^{-1}(y), h^{-1}(y)\right)<\varepsilon(y)\right. \\
&\text { for each } y \in Y, \text { and } h=f \text { on } Y-U\} .
\end{aligned}
$$

A subset $X$ of $Y$ is thin in $Y$ if, for each open $U$ containing $X$ and each map $\varepsilon: U \rightarrow R^{+}$, there exists $h \in V_{U}(1, \varepsilon)$ such that $h(X) \cap X=\varnothing$.

Let $\mathscr{S}$ be some collection of subsets of $Y$, and let $\mathscr{S}_{+}$[resp. $\mathscr{S}_{++}$] be the collection of all finite [resp. countable] unions of closed subsets of elements of $\mathscr{S}$. A subset $B$ of $Y$ is a pseudo-boundary for $\mathscr{S}$ in $Y$ if $B \in \mathscr{S}_{++}$ and the following absorption property holds: For each $S \in \mathscr{S}, U$ open in $Y$, and $\varepsilon: U \rightarrow R^{+}$a map, there exists $h \in V_{U}(1, \varepsilon)$ such that $h(S \cap U) \subset B$. $P=Y-B$ is a pseudo-interior for $\mathscr{S}$ in $Y$.

Possible Axioms for $\mathscr{S}$ : 
I (closed). The elements of $\mathscr{S}$ are closed in $Y$.

II (invariant). The elements of $\mathscr{S}$ are invariant under $H(Y)$.

III (thin). The elements of $\mathscr{S}_{+}$are thin in Y.

TheOREM 2.1 (WeSt [19, TheORem 1]). Let $\mathscr{S}$ satisfy I and II, let $B$ and $B^{\prime}$ be pseudo-boundaries for $\mathscr{S}$ in $Y$, let $U$ be open in $Y$ and let $\varepsilon: U \rightarrow R^{+}$ be a map. Then there exists $f \in V_{U}(1, \varepsilon)$ such that $f(B \cap U)=B^{\prime} \cap U$.

If $B$ is a pseudo-boundary and $T \in \mathscr{S}_{++}$, then $B \cup T$ is also a pseudoboundary. This with Theorem 2.1 leads to

Corollary 2.2. Let $\mathscr{S}$ satisfy I and II, let $\boldsymbol{P}$ be a pseudo-interior for $\mathscr{S}$ in $Y$ and let $T \in \mathscr{S}_{++}$. Then $P \cap T$ is strongly negligible in $P$.

By further considering the implications of Theorem 2.1, one proves

Corollary 2.3. Let $\mathscr{S}$ satisfy I, II and III, and let B be a pseudoboundary for $\mathscr{S}$ in $Y$. If $T \in \mathscr{S}_{+}$, then $B \cap T$ is strongly negligible in $B$. Moreover, if $T^{\prime}$ is any closed subset of $Y$ which lies in $B \mid$ then $T^{\prime}$ is strongly negligible in $B$.

COROLlaRY 2.3 applies in particular to compact subsets of $B$.

3. The universal pseudo-boundaries in $E^{n}$. A closed subset $X$ of a space $Y$ is a $Z_{m}$-set $(m$ an integer $\geqq 0)$ if, for every nonempty $m$-connected open set $U$ in $Y, U-X$ is nonempty and $m$-connected. A closed subset $X$ of $E^{n}$ is a strong $Z_{m}$-set $(-1 \leqq m<n)$ if, for each compact subpolyhydron $P$ of $E^{n}$ having dimension $\leqq m+1$, and each $\varepsilon>0$, there is an $\varepsilon$-push (see [7]) $h$ of $\left(E^{n}, X \cap P\right)$ such that $h(X) \cap P=\varnothing$. Let $\mathscr{M}_{n}^{k}$ be the family of all strong $Z_{n-k-2}$-sets in $E^{n},-1 \leqq k<n$.

EXAMPLES OF STRONG $Z_{n-k-2}$-SETS IN $E^{n}$. Any $k$-dimensional subpolyhedron of $E^{n}$; any compact subset whose complement is 1-ULC, provided $k \leqq n-3$ and $n \neq 4$; if $k=n-1$ or if $1 \neq k=n-2$, then strong $Z_{n-k-2}$ is equivalent to dimension $\leqq k$; strong $Z_{n-k-2}$ always implies $\operatorname{dim} \leqq k$.

Lemma 3.1. $\mathscr{M}_{n}^{k}$ satisfies I and II. If $n \geqq 2 k+1, \mathscr{M}_{n}^{k}$ also satisfies III.

Obviously I holds. The hardest part of showing II is the case $k \leqq n-3$ and $n \geqq 5$ : For this, one uses engulfing theorems in [7], [8] or alternatively [16], to show that strong $Z_{n-k-2}$ is equivalent to $Z_{1}$ and dimension $\leqq k$; the latter is indeed an invariant property. The remaining special cases are derived from [4], [5], [10] and [12]. A general position argument verifies III.

$A$ pseudo-boundary. Partition $E^{1}$ into intervals whose endpoints are $l / 3^{i-1}, i \geqq 1, l$ an integer. Regarding $E^{n}$ as a product, let $J_{i}$ be the product cell complex partitioning $E^{n}$, its $k$-skeleton being $J_{i}^{k}$. Let $K_{i}$ be the sub- 
complex of $J_{i+1}$ generated by the cells which touch cells of $J_{i}^{k}$. Let $B_{i}\left(\mathscr{M}_{n}^{k}\right)=\bigcup_{j=i}^{\infty}\left|K_{j}\right|$ and let $B\left(\mathscr{M}_{n}^{k}\right)=\bigcup_{i=1}^{\infty} B_{i}\left(\mathscr{M}_{n}^{k}\right)$. The intersection of $B_{i}\left(\mathscr{M}_{n}^{k}\right)$ with an $n$-cell of $J_{i}$ is often called a Menger universal $k$-dimensional compactum in $E^{n}$ (its universality, which we do not need, is proved in [17]). We therefore call $B\left(\mathscr{M}_{n}^{k}\right)$ the universal k-dimensional pseudoboundary in $E^{n}$.

TheOREM 3.2. $B\left(\mathscr{M}_{n}^{k}\right)$ is a pseudo-boundary for $\mathscr{M}_{n}^{k}$ in $E^{n}$.

Clearly $B\left(\mathscr{M}_{n}^{k}\right) \in\left(\mathscr{M}_{n}^{k}\right)_{++}$. By a method of Bothe [6], each element of $\mathscr{M}_{n}^{k}$ can be (ambiently) pushed into $B_{i}\left(\mathscr{M}_{n}^{k}\right)$ for any $i$. Bothe's methods can be adapted to show that $B\left(\mathscr{M}_{n}^{k}\right)$ has the absorption property as required.

Besides Theorem 1.1 (in which one takes $B^{k}=B\left(\mathscr{M}_{n}^{k}\right)$ and $P^{n-k-1}=$ $\left.E^{n}-B\left(\mathscr{M}_{n}^{k}\right)=P\left(\mathscr{M}_{n}^{k}\right)\right)$ we have other interesting negligibility theorems which reflect the situation in $I^{\omega}$ (compare Theorems 0 and 1 of [2]).

Theorem 3.3. Assume $(n, k) \neq(3,1),(4,0)$ or $(4,1)$. A closed subset of $P\left(\mathscr{M}_{n}^{k}\right)$ is strongly negligible in $P\left(\mathscr{M}_{n}^{k}\right)$ if and only if it is a $Z_{n-k-2}$-set in $P\left(\mathscr{M}_{n}^{k}\right)$. An arbitrary subset is strongly negligible if and only if it is the countable union of $Z_{n-k-2}$-sets.

TheOREM 3.4. $A$ closed subset of $B\left(\mathscr{M}_{2 k+1}^{k}\right), k \neq 1$, is strongly negligible in $B\left(\mathscr{M}_{2 k+1}^{k}\right)$ if and only if it is a $Z_{k-1}$-set in $B\left(\mathscr{M}_{2 k+1}^{k}\right)$.

Note that Theorems 3.3 and 3.4 are "intrinsic" theorems: $E^{n}$ is nowhere mentioned.

4. The polyhedral pseudo-boundaries in $E^{n}, n \neq 4$. Let $\mathscr{P}_{n}^{k}$ be the family of all tame $k$-dimensional polyhedra in $E^{n}$. From $\S 3$, we deduce

Lemma 4.1. $\mathscr{P}_{n}^{k}$ satisfies I and II. If $n \geqq 2 k+1, \mathscr{P}_{n}^{k}$ also satisfies III.

The polyhedral $k$-dimensional pseudo-boundary in $E^{n}$ is the set $\widetilde{B}_{n}^{k}$ defined in $\S 1$. Here we will call it $B\left(\mathscr{P}_{n}^{k}\right)$. We have

THEOREM 4.2. If $n \neq 4, B\left(\mathscr{P}_{n}^{k}\right)$ is a pseudo-boundary for $\mathscr{P}_{n}^{k}$ in $E^{n}$.

To prove Theorem 4.2 one needs the "Hauptvermutung" for $E^{n}$ ([4], [11] and [14]) which is unknown when $n=4$.

The negligibility Theorem 1.2 follows as in $\S 2$, though $1.2(4)$ seems to require a codimension 3 taming theorem (see Theorem 4 of [9] and Theorem 1 of [15]).

5. The infinite-dimensional analogy. The usual pseudo-boundary of $I^{w}$ is itself the countable union of copies of $I^{\omega}$; in other words, it is the coun- 
ble union of universal compacta in $I^{\omega}$. Compare with the universal $k$-dimensional pseudo-boundary $B\left(\mathscr{M}_{n}^{k}\right)$ of $\S 3$ which is the countable union of universal $k$-dimensional compacta in $E^{n}$. There is also a smaller pseudo-boundary in $I^{\omega}$, defined by Anderson in [2]. This one is the countable union of finite-dimensional cubes. Compare with the polyhedral $k$-dimensional pseudo-boundary $B\left(\mathscr{P}_{n}^{k}\right)$ of $\$ 4$ which is the countable union of $k$-dimensional cubes. So far a good analogy.

But more is known about the infinite-dimensional case. While the two pseudo-boundaries in $I^{\omega}$ are not equivalent (in one case take $\mathscr{S}$ to be the family of all $Z$-sets in $I^{\omega}$, in the other take $\mathscr{S}$ to be the family of all tame polyhedra in $\left.I^{\omega}\right)$, the corresponding pseudo-interiors are both homeomorphic to Hilbert space $l_{2}$ (see [2]). Letting $P\left(\mathscr{M}_{n}^{k}\right)=E^{n}-B\left(\mathscr{M}_{n}^{k}\right)$ and $P\left(\mathscr{P}_{n}^{k}\right)=E^{n}-B\left(\mathscr{P}_{n}^{k}\right)$, the analogy suggests

CONJECTURE. If $n \leqq 2 k+1$, then $P\left(\mathscr{M}_{n}^{k}\right)$ and $P\left(\mathscr{P}_{n}^{k}\right)$ are homeomorphic.

The conjecture is easily proved when $k=0$ and $n=1$. R. D. Anderson has pointed out that it is false if $k=0$ and $n \geqq 2$. The restriction $n \leqq 2 k+1$ seems reasonable.

6. Pseudo-boundaries in topological manifolds. Let $M$ be a separable metrizable $n$-manifold without boundary. A euclidean chart for $M$ is a pair $(h, W)$ where $W$ is open in $M$ and $h: E^{n} \rightarrow W$ is a homeomorphism. A closed subset $X$ of $M$ is a local strong $Z_{n-k-2}$-set in $M$ (resp. local tame $k$-dimensional polyhedral set in $M$ ) if for each $x \in X$ there is a euclidean chart $(h, W)$ with $x \in W$ and $h^{-1}(X)$ a strong $Z_{n-k-2}$-set in $E^{n}$ (resp. a tame $k$-dimensional polyhedron in $E^{n}$ ). Let $\mathscr{M}_{M}^{k}$ (resp. $\mathscr{P}_{M}^{k}$ ) be the family of all local strong $Z_{n-k-2}$-sets in $M$ (resp. all local tame $k$-dimensional polyhedral sets in $M$ ).

Lemma 6.1. $\mathscr{M}_{M}^{k}$ and $\mathscr{P}_{M}^{k}$ satisfy I and II; if $n \geqq 2 k+1$ and $(n, k) \neq(4,0)$ or $(4,1)$, they also satisfy III.

Let $\left\{\left(h_{i}, W_{i}\right)\right.$ be a countable set of euclidean charts such that $M=$ $\bigcup_{i=1}^{\infty} W_{i}$. Define $B\left(\mathscr{M}_{M}^{k}\right)=\bigcup_{i=1}^{\infty} h_{i}\left(B\left(\mathscr{M}_{n}^{k}\right)\right)$ and $B\left(\mathscr{P}_{M}^{k}\right)=\bigcup_{i=1}^{\infty}\left(B\left(\mathscr{P}_{n}^{k}\right)\right)$.

THEOREM 6.2. If $(n, k) \neq(4,0)$ or $(4,1)$, then $B\left(\mathscr{M}_{M}^{k}\right)$ is a pseudoboundary for $\mathscr{M}_{M}^{k}$ in $M$. If $n \neq 4, B\left(\mathscr{P}_{M}^{k}\right)$ is a pseudo-boundary for $\mathscr{P}_{M}^{k}$ in $M$.

2.1, 6.1 and 6.2 imply that $B\left(\mathscr{M}_{M}^{k}\right)$ and $B\left(\mathscr{P}_{M}^{k}\right)$ are independent of the charts $\left\{\left(h_{i}, W_{i}\right)\right\}$ up to homeomorphism of $M$.

Thus the notions of universal and polyhedral pseudo-boundaries can be sensibly extended to manifolds. Negligibility theorems follow as in previous sections. 


\section{REFERENCES}

1. R. D. Anderson, Strongly negligible sets in Fréchet manifolds, Bull. Amer. Math. Soc. 75 (1969), 64-67. MR 38 \# 6634.

2. - On sigma-compact subsets of infinite-dimensional spaces, Trans. Amer. Math. Soc. (to appear).

3. C. Bessaga and A. Pełczynski, The estimated extension theorem, homogeneous collections and skeletons and their applications to the topological classification of linear metric spaces and convex sets, Fund. Math. 69 (1970), 153-190. MR 42 \#8227. 816.

4. R. H. Bing, Locally tame sets are tame, Ann. of Math. (2) 59 (1954), 145-158. MR 15,

5. R. H. Bing and J. M. Kister, Taming complexes in hyperplanes, Duke Math. J. 31 (1964), 491-511. MR 29 \#1626.

6. H. G. Bothe, Eine Einbettung $m$-dimensionaler Mengen in einen $(m+1)$-dimensionalen absoluten Retrakt, Fund. Math. 52 (1963), 209-224. MR 27 \# 1953.

7. J. L. Bryant, On embeddings of compacta in Euclidean space, Proc. Amer. Math. Soc. 23 (1969), 46-51. MR 39 \#6286.

8. 265-270. MR 43 \# 1154.

9. A. V. Cernavskiǔ, Topological imbeddings of manifolds, Dokl. Akad. Nauk SSSR 187 (1969), 1247-1250 = Soviet Math. Dokl. 10 (1969), 1037-1041. MR 41 \# 4547.

10. H. Gluck, Embeddings in the trivial range, Ann. of Math. (2) 81 (1965), 195-210. MR 30 \# 3456.

11. R. Kirby, Lectures on triangulations of manifolds, Mimeographed notes, University of California, Los Angeles, Calif., 1969.

12. D. R. McMillan, Jr., Taming Cantor sets in $E^{n}$, Bull. Amer. Math. Soc. 70 (1964), 706-708. MR 29 \#1628.

13. K. Menger, Über umfassendste n-dimensionale Mengen, Nederl. Akad. Wetensch. Proc. Ser. A 29 (1926), 1125-1128.

14. T. Rado, Uber den Begriff der Riemannschen Fläche, Acta Sci. Math. Szeged. 2 (1925), 101-121.

15. T. B. Rushing, Taming codimension three embeddings, Bull. Amer. Math. Soc. 75 (1969), 815-820. MR 39 \#6328.

16. M. A. Stan'ko, Imbedding of compacta in Euclidean space, Mat. Sb. 83 (125) (1970), 234-255 = Math. USSR Sb. 12 (1970), 234-254. MR 42 \#6804.

17. Solution to Menger's problem in the class of compacts, Dokl. Akad. Nauk SSSR 201 (1971), 1846-1849 = Soviet Math. Dokl. 12 (1971), 1299-1301.

18. H. Torunczyk, Skeletonized sets in complete metric spaces and homeomorphisms of the Hilbert cube, Bull. Acad. Polon. Sci. Sér. Sci. Math. Astronom. Phys. 18 (1970), 119-126. MR 41 \#9194.

19. J. E. West, The ambient homeomorphy of an incomplete subspace of infinite-dimensional Hilbert spaces, Pacific J. Math. 34 (1970), 257-268. MR 43 \# 2748.

School of Mathematics, Institute for Advanced Study, Princeton, New Jersey 08540

Current address (Ross Geoghegan): Department of Mathematics, State University of New York, Binghamton, New York 13901

Current address (R. Richard Summerhill): Department of Mathematics, Kansas State University, Manhattan, Kansas 66502 